\title{
Embedding Metric Spaces in the Rectilinear Plane: a Six-Point Criterion
}

\author{
H.-J. Bandelt ${ }^{1}$ and V. Chepoi ${ }^{2}$ \\ ${ }^{1}$ Mathematisches Seminar, Universität Hamburg, \\ Bundesstrasse 55, D-20146 Hamburg, Germany \\ 2 Catedra de cibematică matematică, Universitatea de stat din Moldova, \\ str. A. Mateevici 60, 277009 Chişinău, Moldova \\ chepoi@university.moldova.su.uunet
}

\begin{abstract}
We show that a metric space embeds in the rectilinear plane (i.e., is $L^{1}$ embeddable in $\mathbb{R}^{2}$ ) if and only if every subspace with five or six points does. A simple construction shows that for higher dimensions $k$ of the host rectilinear space the number $c(k)$ of points that need to be tested grows at least quadratically with $k$, thus disproving a conjecture of Seth and Jerome Malitz.
\end{abstract}

\section{Introduction}

Recently, Malitz and Malitz [7] have proven a Menger-type theorem for the rectilinear plane $\mathbb{R}^{2}$ (endowed with the $L^{1}$-metric): a metric space is embeddable in the plane if and only if every subspace with at most 11 points is such. They suspected that it would even suffice to consider the subspaces having no more than six points but their method of proof cannot settle this stronger assertion. In contrast to the two-dimensional case, no corresponding result bounding the size of the subspaces to be tested is known for $L^{\prime}$-embeddability in $\mathbb{R}^{k}$ (the $k$-dimensional rectilinear space) when $k \geq 3$. It was further conjectured in [7] that $c(k)=2 k+2$ is the smallest number such that every metric space embeds in the rectilinear space $\mathbb{R}^{k}$ whenever all subspaces with at most $c(k)$ points embed.

In this note we contribute to the two conjectures. First, the bad news: if $c(k)$ exists, then

$$
c(k) \geq k^{2} \quad \text { for } \quad k \geq 3 \text { odd }
$$


In particular, $c(3) \geq 9$ holds, thus exceeding the value 8 predicted in [7]; a further example shows that $c(3)$ must even exceed 9. The good news concerns the two-dimensional case, confirming $c(2)=6$ :

Theorem A. A metric space embeds in the rectilinear plane if every subspace of size at most 6 does.

The two-dimensional case is really special in that we can take advantage of the decomposition theory for finite metric spaces developed by Bandelt and Dress [2]. Every finite subspace of the rectilinear plane is "totally decomposable" [2]. This property entails embeddability in some higher-dimensional rectilinear space and is fully described by a five-point condition. The structure of a totally decomposable space is completely determined by the combinatorial properties of its ordered system of half-spaces, so that we can actually confine ourselves to (unweighted) graphs. Guided by van de Vel's Theory of Convex Structures [12] we can readily discern the required grid embedding by checking a number of forbidden six-point configurations.

\section{Total Decomposability}

The ingredients of the decomposition theory [2] are the following. Let $(X, d)$ be a finite metric space, where however $d(x, y)=0$ need not imply $x=y$. For any nonempty subsets $A$ and $B$ of $X$ define the isolation index by

$$
\begin{gathered}
\alpha_{A . B}=\frac{1}{2} \cdot \min _{\substack{a, a^{\prime} \in A \\
b . b^{\prime} \in B}}\left(\operatorname { m a x } \left\{d(a, b)+d\left(a^{\prime}, b^{\prime}\right), d\left(a, b^{\prime}\right)+d\left(a^{\prime}, b\right), d\left(a, a^{\prime}\right)\right.\right. \\
\left.\left.+d\left(b, b^{\prime}\right)\right\}-d\left(a, a^{\prime}\right)-d\left(b, b^{\prime}\right)\right) .
\end{gathered}
$$

The unordered pair $\{A, B\}$ is a split if it constitutes a partition of $X$ into two nonempty parts. It is said to be a $d$-split exactly when $\alpha_{A, B}>0$. Either part $A, B$ is then called a $d$-half-space of $X$. The space $(X, d)$ is called totally decomposable if

$$
d=\sum \alpha_{A, B} \cdot \delta_{A, B},
$$

where the sum extends over all $d$-splits and $\delta_{A, B}$ denotes the split metric associated to $\{A, B\}$ :

$$
\delta_{A, B}(x, y)= \begin{cases}0 & \text { if } x, y \in A \text { or } x, y \in B, \\ 1 & \text { otherwise. }\end{cases}
$$

Note that $d$-splits are necessarily weakly compatible splits, in the sense that there are no three of them, $\left\{A_{1}, B_{1}\right\},\left\{A_{2}, B_{2}\right\},\left\{A_{3}, B_{3}\right\}$, together with three points $x_{1}, x_{2}, x_{3}$ such that $x_{i} \in A_{j}$ exactly when $i \neq j$. Now, departing from any collection of weakly compatible splits of the set $X$, any positive linear combination of the associated split metrics yields a totally decomposable metric $d$ on $X$ such that the $d$-splits are exactly the splits from the given collection [2]. 
Fact 1 [2]. A finite metric space $(X, d)$ is totally decomposable if and only if

$$
\alpha_{\{t, u\},\{v, w\}}=\alpha_{\{t, u, x\},\{v, w\}}+\alpha_{\{t, u\},\{v, w, x\}}
$$

for all $t, u, v, w, x \in X$.

Particular instances of totally decomposable spaces are tree spaces, i.e., finite metric subspaces of dendrons (the latter being compact connected spaces in which any two distinct points get separated by removing some third point). Whether or not a totally decomposable space is a tree space can be read off from the $d$-splits. Two splits $\{A, B\}$ and $\{C, D\}$ are said to be compatible if one of the four intersections $A \cap C, A \cap D$, $B \cap C$, and $B \cap D$ is empty; otherwise, the splits are called incompatible. The tree spaces are exactly the totally decomposable spaces in which any two $d$-splits are compatible [2, Corollary 7]. In general, the compatibility graph of the space has the $d$-splits as its vertices and pairs of compatible $d$-splits as its edges; the graph complementary to the former is called the incompatibility graph. The totally decomposable spaces with bipartite incompatibility graphs are exactly those which embed in the product of two dendrons. A characterization in terms of forbidden configuration is recorded next.

Fact 2 [2]. A finite metric space $(X, d)$ embeds in the product of two dendrons if and only if it is totally decomposable and satisfies the following two conditions:

(1) There is no six-point subspace $Y=\left\{y_{0}, \ldots, y_{5}\right\}$ such that $\left\{\left\{y_{i}, y_{i+1}, y_{i+2}\right\}\right.$, $\left.\left\{y_{i+3}, y_{i+4}, y_{i+5}\right\}\right\}(i=0,1, \ldots, 5$; indices modulo 6$)$ are $d$-splits of $Y$.

(2) There is no $(2 p+1)$-point subspace $Z=\left\{z_{0}, \ldots, z_{2 p}\right\}(p \geq 2)$ such that $\left\{\left\{z_{i}, z_{i+1}\right\}, Z-\left\{z_{i}, z_{i+1}\right\}\right\}(i=0,1, \ldots, 2 p$; indices modulo $2 p+1)$ are $d$-splits of $Z$.

This fact suggests that there is no $n$-point criterion for embeddability in the product of two dendrons. Indeed, consider the space $X$ with $2 p+1$ points $x_{0}, \ldots, x_{2 p}(p \geq 2)$ such that the distance $d$ between $x_{i}$ and $x_{j}$ is 2 if $i$ and $j$ differ by 1 modulo $2 p+1$ and equals 4 otherwise. This space is totally decomposable having $\left\{\left\{x_{i}, x_{i+1}\right\}, X-\left\{x_{i}, x_{i+1}\right\}\right\}$ $(i=0, \ldots, 2 p$; indices modulo $2 p+1)$ as $d$-splits with isolation indices equal to 1 . Removing a single point from $X$ results in a space that embeds in the product of two dendrons (each having $p$ endpoints). It is conceivable that bounding the number of endpoints of the dendrons under consideration could lead to some $n$-point criterion for embeddability. A necessary condition that might be of use in this respect is the following observation.

Lemma 1. Let $(X, d)$ be a finite subspace of the product of two dendrons having $p$ and $q$ endpoints, respectively. Then for any choice of $n \leq p+q+1$ distinct $d$-splits $\left\{A_{1}, B_{1}\right\}, \ldots,\left\{A_{n}, B_{n}\right\}$ the collection of half-spaces $A_{1}, \ldots, A_{n}, B_{1}, \ldots, B_{n}$ ordered by inclusion has at most $p+q$ minimal members.

Proof. Projecting $X$ onto the two factors (dendrons $D_{1}$ and $D_{2}$ ) yields two tree spaces $\left(X, d_{1}\right)$ and $\left(X, d_{2}\right)$ with $d=d_{1}+d_{2}$. Every $d$-split is then either a $d_{1}$-split or a $d_{2}$-split, 
and vice versa [2, Corollary 8]. Consider the metric $d^{\prime}$ obtained from $d$ by removing all $d$-splits that are not among $\left\{A_{1}, B_{1}\right\}, \ldots,\left\{A_{n}, B_{n}\right\}$, i.e.,

$$
d^{\prime}=\sum_{i=1}^{n} \alpha_{A_{i}, B_{i}} \cdot \delta_{A_{i}, B_{i}}
$$

The metric space $\left(X, d^{\prime}\right)$ embeds in the product of two dendrons $D_{1}^{\prime}$ and $D_{2}^{\prime}$ obtained from $D_{1}$ and $D_{2}$ by contradicting some segments (namely, those corresponding to the eliminated splits). $D_{1}^{\prime}$ and $D_{2}^{\prime}$ have at most $p$ and $q$ endpoints, respectively. The projections of $\left(X, d^{\prime}\right)$ to $D_{1}^{\prime}$ and $D_{2}^{\prime}$ yield tree metrics $d_{1}^{\prime}$ and $d_{2}^{\prime}$ with sum $d^{\prime}$ such that $\left\{A_{1}, B_{1}\right\}, \ldots,\left\{A_{n}, B_{n}\right\}$ comprise all $d_{1}^{\prime}$-splits and $d_{2}^{\prime}$-splits taken together. Since among the $d_{1}^{\prime}$-half-spaces (or the $d_{2}^{\prime}$-half-spaces, respectively) we can have at most $p$ (or $q$, respectively) minimal half-spaces, there are at most $p+q$ minimal members of the ordered set of half-spaces $A_{1}, \ldots, A_{n}, B_{1}, \ldots, B_{n}$.

The preceding proof indicates that a number of properties of a totally decomposable space do not depend on the actual (positive) values of the isolation indices of its splits. If $d$ is composed as described in (*), we may consider the integral metric $\delta$ defined by

$$
\delta=\sum_{d-s p l i s\{A, B\}} \delta_{A, B}
$$

which has the same splits as $d$. If the number of $d$-splits is $m$ in this case, then we can embed the integral space $(X, \delta)$ in an $m$-dimensional cube $Q$. In order to represent the $d$ splits graphically, it typically suffices to employ only a minor part of $Q$. A median graph $G$ is a connected graph in which for each triple $u, v, w$ of vertices a unique ("median") vertex exists that simultaneously lies on shortest paths between any two vertices from $u, v, w$ (see [3] and [12]). A connected induced subgraph $F$ of $G$ is called a median subgraph of $G$ if for any three vertices of $F$ their "median" vertex in $G$ belongs to $F$. For the above graph-theoretic representation of the $d$-splits of $(X, d)$ it suffices to consider the smallest median subgraph of the $m$-cube $Q$ that includes $X$. This graph, referred to as the median graph generated by the integral space $(X, \delta)$, can be constructed directly from the collection of $d$-splits of $X$; see [1]. The $d$-splits of $X$ then extend to the pairs of complementary half-spaces of $G$; a half-space of $G$ is a set $H$ of vertices that includes every shortest path of $G$ between any two vertices of $H$ and is disjoint from any shortest path of $G$ between two points outside $H$. That $(X, \delta)$ generates $G$ is reflected by the intersection pattern of the half-spaces of $G$ : any nonempty intersection of two half-spaces of $G$ contains some point of $X[1]$. Observe that $(X, d)$ embeds in a product of two dendrons (with $p$ and $q$ endpoints, respectively) if and only if $G$ embeds in a Cartesian product of two trees (with $p$ and $q$ end vertices, respectively). Recall that in a Cartesian product of graphs two vertex-tuples are adjacent when they are adjacent in exactly one coordinate and identical otherwise [12]. We summarize the consequences of the preceding discussion in the following lemma.

Lemma 2. Let $(X, d)$ be a totally decomposable space, and let $G$ be the median graph generated by the associated integral space $(X, \delta)$. The embedding of $X$ into $G$ induces 
isomorphisms

(i) between the ordered sets of $d$-half-spaces of $X$ and half-spaces of $G$ and

(ii) between the incompatibility graphs of the $d$-splits of $X$ and the splits consisting of complementary half-spaces of $G$.

Moreover, $(X, d)$ embeds in a rectilinear plane exactly when $G$ embeds (as a median subgraph) in a grid (i.e., a Cartesian product of two paths).

\section{A Structural Characterization of Subspaces of the Plane}

The structural result paving the way to Theorem A characterizes the totally decomposable spaces that embed in the rectilinear plane in terms of forbidden configurations of halfspaces:

Theorem B. A totally decomposable space $(X, d)$ embeds in the rectilinear plane if and only iffor any $d$-splits $\left\{A_{1}, B_{1}\right\}, \ldots,\left\{A_{n}, B_{n}\right\}(n \leq 5)$ the ordered set of half-spaces $A_{1}, \ldots, A_{n}, B_{1}, \ldots, B_{n}$ has at most four minimal members.

Necessity is covered by Lemma 1. To prove the converse, we proceed by induction on the number of $d$-splits. As discussed in the preceding section we may pass from the metric $d$ to the associated integral metric $\delta$ given by $(* *)$, in order to decide the embeddability question. In view of Lemma 2 we may thus replace $(X, d)$ by the median graph. $G$ that is generated by $(X, \delta)$. Then, by virtue of the induction hypothesis, every maximal half-space $F$ of $G$ embeds in a (two-dimensional) grid, that is, the Cartesian product of two paths. This grid has at most four distinct minimal half-spaces, each constituting a boundary path. This property is inherited by its median subgraph $F$.

Lemma 3. If a median subgraph $F$ of a grid has at most three distinct minimal halfspaces, then it has a pendant vertex $v$ (such that the vertex-deleted subgraph $F-v$ is included in a smaller grid).

Proof. The grid in which $F$ embeds may be assumed as small as possible. Then each of the boundary paths of the grid includes a minimal half-space of $F$. In the case that not all of these half-spaces are different, then at least one of them is a singleton $\{v\}$ (so that $v$ is a corner vertex of the grid). Then $F-v$ lies in a subgrid obtained from the given grid by removing one boundary path.

The obvious strategy to establish the grid embedding of $G$ is first to embed a suitable maximal half-space $F$ of $G$ in a grid and to extend the grid accordingly by attaching a new boundary path. Three main cases have then to be distinguished.

Case 1: Removing some minimal half-space $H$ from $G$ results in a median graph $G-H$ having four distinct minimal half-spaces $P_{1}, P_{2}, P_{3}, P_{4}$. If each of the (distinct) halfspaces of $G$ extending $P_{1}, P_{2}, P_{3}, P_{4}$, respectively, is incomparable with $H$, then we would 

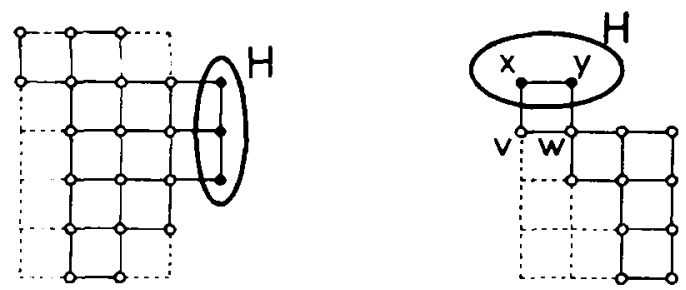

Fig. 1. Extending the grid embedding in Cases 1 and 2.

obtain five distinct minimal half-spaces in $G$, contrary to the hypothesis. Consequently, some $P_{i}$ extends to a half-space of $G$ that includes $H$. The neighbors of the vertices of $H$ in $G$ then induce a subpath of $P_{i}$ (isomorphic to $H$ ). Since $P_{i}$ is located on the boundary of the grid, we can simply extend the grid by a new boundary path containing $H$ as a subpath; see Fig. 1. This settles Case 1.

In what follows we may therefore assume that every maximal half-space of $G$ constitutes a median subgraph $F$ of a grid such that $F$ has at most three distinct minimal half-spaces. We then choose a half-space $H$ of $G$ with the smallest number of vertices.

Case 2: The half-space $H$ of minimum size has at least two vertices. Since $G-H$ has some pendant vertex $v$ by Lemma 3 and thus possesses a singleton half-space, we infer that $H$ contains a neighbor $x$ of $v$ (for otherwise, $\{v\}$ would also constitute a half-space of $G$, contrary to the choice of $H$ ). Then $\{v, x\}$ is a half-space of $G$, whence $H$ consists of exactly two adjacent vertices, $x$ and $y$, so that $y$ is adjacent to the unique neighbor $w$ of $v$ in $G-H$. The vertices $v, x, y, w$ thus induce a 4-cycle intersecting with the grid in an edge of the boundary. The grid can then be extended to incorporate this cycle as well; see Fig. 1.

Case 3: The half-space $H$ is a singleton. Removing $H$ yields a median subgraph of a grid that again has a pendant vertex. Successively dropping pendant vertices eventually results in either a singleton or in a median subgraph $F$ of some grid such that $F$ has four distinct minimal half-spaces $P_{1}, P_{2}, P_{3}, P_{4}$, each being paths of length at least 1 located on the boundary of the grid. In the former case $G$ is a tree which cannot have more than four end vertices (according to the hypothesis on $G$ ); clearly such a tree embeds in a grid. Therefore assume the latter case: $G$ consists of $F$ and a number of pairwise disjoint trees $T_{1}, \ldots, T_{m}$ emanating from $F$, that is, each having exactly one vertex in common with $F$, the "root" of the respective tree. Each root $z$ must belong to one of the paths $P_{1}, \ldots, P_{4}$; indeed, otherwise the following five pairs of complementary halfspaces would violate the hypothesis: a tip $t$ (i.e., an end vertex different from the root) of the tree $T_{i}$ incident with $z$, the complement $G-t$, and the half-spaces of $G$ extending $P_{1}, \ldots, P_{4}$, respectively, and their complements. More generally, if we select any subset of $k$ tips from the rooted trees $T_{1} \ldots, T_{m}$, the total number of paths among $P_{1}, \ldots, P_{4}$ that are incident with the root of a tree from which a tip was selected must be at least $k$. Thus, Hall's condition of the matching theorem is satisfied, and we can match the tips to the paths $P_{1}, \ldots, P_{4}$ (connected via the trees). In particular, $m \leq 4$ holds, each tree $T_{i}$ has at most two tips, and in case $T_{i}$ has two tips the root must be located on a 

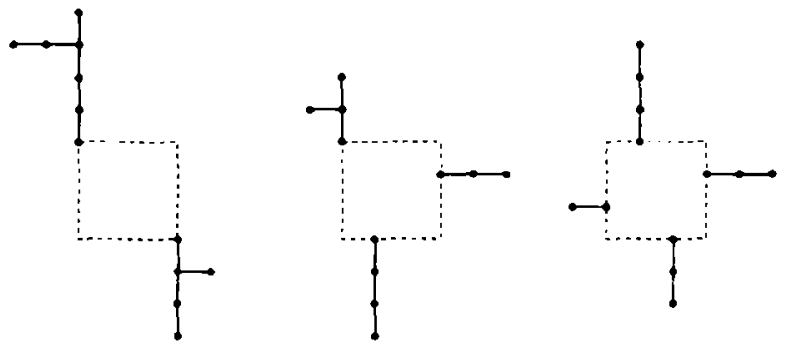

Fig. 2. Extending the grid embedding for each of the three generic instances in Case 3.

corner vertex of the grid, being incident with two paths from $P_{1}, \ldots, P_{4}$. When a tip $t$ is matched to a path $P_{i}$, we let the terminal path of the tree leading to $t$ be orthogonal to $P_{i}$ in order to achieve a grid embedding (where we have to deal with three generic situations according to the number of trees with two tips); see Fig. 2. This concludes the proof of Theorem B.

\section{Proof of Theorem A}

It suffices to prove the theorem for finite metric spaces $(X, d)$ according to [7]. Our proof of the six-point criterion assumes finiteness but otherwise does not make use of the eleven-point criterion established in [7]. First note that the five-point condition recorded in Fact 1 guarantees total decomposability. We thus have to check that any violation of the half-space condition in Theorem B could be detected within some subspace of at most six points.

Assume that the $d$-splits $\left\{A_{1}, B_{1}\right\}, \ldots,\left\{A_{n}, B_{n}\right\}(n \leq 5)$ violate the condition of Theorem $\mathrm{B}$. We distinguish three cases.

Case 1: $n=3$. Since among the six half-spaces five are minimal, so are all six. Hence the three $d$-splits are pairwise incompatible. The median graph $G$ generated by the integral space $(X, \delta)$ must contain a 3-cube that is cut by each of the three $d$-splits (see [11] and [12]). Consider the coarsest partition of $X$ refining the three given $d$-splits. Each block of this partition consists of the points from $X$ closest to one vertex of the 3-cube, their "gate" in the 3-cube. Now the 3-cube can be partitioned into two (complementary) sets of four pairwise nonadjacent vertices. Since $d$-splits are weakly compatible, at least one vertex from either set does not occur as a gate of the blocks. These selected vertices cannot be adjacent, for, otherwise, two of the given three $d$-splits would be compatible. Hence these vertices are opposite (diametrical) in the 3-cube, and thus the remaining six vertices form a 6-cycle and constitute the gates of six nonempty blocks of $X$. Selecting one point from each block then yields the forbidden six-point configuration (1) from Fact 2.

Case 2: $n=4$. Then the half-spaces of at least one $d$-split, $\left\{A_{4}, B_{4}\right\}$ say, are both minimal among the eight half-spaces $A_{1}, \ldots, A_{4}, B_{1}, \ldots, B_{4}$. Hence the split $\left\{A_{4}, B_{4}\right\}$ is incompatible with the other three splits $\left\{A_{i}, B_{i}\right\}$. In view of Case 1 we cannot have 
three pairwise incompatible $d$-splits. Therefore the splits $\left\{A_{1}, B_{1}\right\},\left\{A_{2}, B_{2}\right\},\left\{A_{3}, B_{3}\right\}$ are pairwise compatible, whence each of them comprises a half-space, $A_{i}(i=1,2,3)$ say, which is minimal among the eight half-spaces. Then, necessarily, $A_{1}, A_{2}, A_{3}$ are pairwise disjoint, whereas each intersection $A_{i} \cap A_{4}$ and $A_{i} \cap B_{4}(i=1,2,3)$ is nonempty. Selecting a point of $X$ from these six intersections yields a six-point subspace that violates Theorem B.

Case 3: $n=5$. We may assume that the minimal members among $A_{1}, \ldots, A_{5}$, $B_{1}, \ldots, B_{5}$ are exactly the half-spaces $A_{1}, \ldots, A_{5}$ (for otherwise, we are back in one of the previous cases). The incompatibility graph of the five splits $\left\{A_{i}, B_{i}\right\}$ is isomorphic to the intersection graph of the half-spaces $A_{1}, \ldots, A_{5}$. It is triangle-free by Case 1 and has maximum degree at most 2 by virtue of Case 2 . Hence the graph is a disjoint union of paths and possibly one cycle. For each half-space $A_{i}$ of degree at most 1 in the graph we can select a point from $A_{i}$ that does not belong to any other half-space $A_{j}$. Moreover, we select one point from each nonempty intersection $A_{j} \cap A_{k}(j \neq k)$ for which at least one of $A_{j}, A_{k}$ has degree 2 . The selected points altogether constitute a five- or six-point set $Y$ on which the half-spaces show the same intersection pattern, so that the $A_{i} \cap Y$ $(i=1, \ldots, 5)$ are minimal among the ten half-spaces restricted to $Y$.

This completes the proof of Theorem A.

\section{Forbidden Minors}

The preceding proof also entails the necessary information about all critical spaces, i.e., those which cannot be embedded in the rectilinear plane but all of which proper subspaces are embeddable. Since the decomposition of all five-point spaces as well as all totally decomposable six-point spaces is known [2], the critical spaces could easily be described in terms of their components. Of particular interest are those critical spaces for which the collection of splits is minimal with respect to inclusion. This leads to the following concept of "minor." Every metric $d$ on the finite set $X$ has a unique decomposition into a "split-prime" metric $d_{0}$ (admitting no $d_{0}$-splits) and a positive linear combination of split metrics $\delta_{i}=\delta_{A_{1}, B_{1}}[2]$ :

$$
d=\alpha_{0} d_{0}+\alpha_{1} \delta_{1}+\cdots+\alpha_{n} \delta_{n} \quad\left(\alpha_{0}=1 \text { and } \alpha_{i}>0 \text { for } i \geq 1\right) .
$$

We say that $\left(X, d^{\prime}\right)$ is a minor of $(X, d)$ if $d^{\prime}$ is obtained from $d$ by decreasing the weights of some summands in $(+)$, that is,

$$
d^{\prime}=\beta_{0} d_{0}+\beta_{1} \delta_{1}+\cdots+\beta_{n} \delta_{n} \quad\left(0 \leq \beta_{i} \leq \alpha_{i} \text { for } i=0, \ldots, n\right) .
$$

Two minors can be considered to be equivalent if they possess the same splits. Note that a minor $\left(X, d^{\prime}\right)$ of a finite subspace $(X, d)$ of the rectilinear plane also embeds in the plane. Indeed, this embedding can be realized by successively contracting (finitely many) horizontal or vertical strips in the plane to lines. From [2] we infer that every finite metric space which is not totally decomposable has a minor equivalent to the metric space given by the graph $K_{2.3}$ (first graph of Fig. 3) with respect to the shortest-path metric, which 

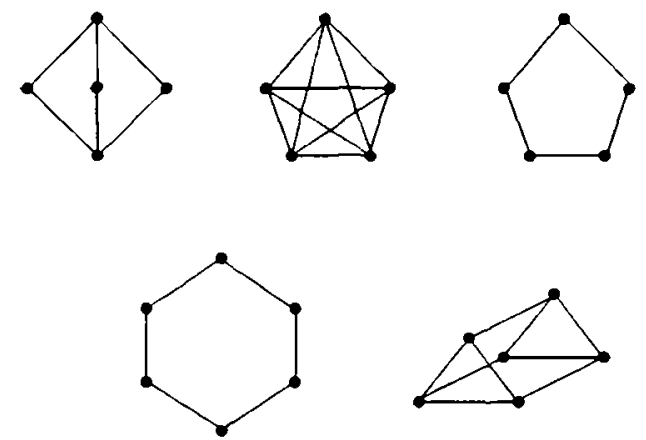

Fig. 3. The five graphs constituting forbidden minors.

cannot be embedded in any rectilinear space. According to the proof of Theorem $A$, the forbidden (totally decomposable) minors with at most four $d$-splits are equivalent to the 6-cycle and the Cartesian product of two complete graphs with two and three vertices, respectively. There are six forbidden minors with five $d$-splits (see Case 3 above): two of them can be represented by graphs, namely, the 5-cycle and the complete graph with five vertices, whereas the remaining four minors (displayed in Fig. 4 as subspaces of median graphs) are nongraphic.

\section{Embedding in $k$-Dimensional Space}

For a certain (rather narrow) class of metric spaces embeddability in $\mathbb{R}^{k}$ can be decided very easily, yet this class is large enough to comprise critical spaces confirming $c(k) \geq k^{2}$ for odd $k$.

The depth of a totally decomposable space $(X, d)$ is the largest number of $d$-halfspaces that are pairwise comparable with respect to inclusion. The depth of a median graph (not necessarily totally decomposable) was defined in [4]. Note that the depth of $(X, d)$ then coincides with the depth of the median graph generated by the corresponding integral space $(X, \delta)$.
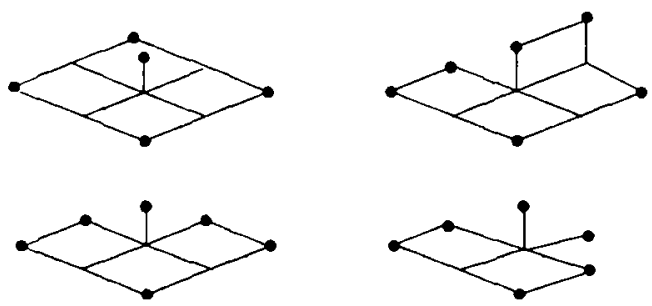

Fig. 4. The four nongraphical forbidden minors (being embedded in three-dimensional space). 
Lemma 4. Let $(X, d)$ be a totally decomposable space of depth at most 2 having exactly $q$ distinct $d$-splits. If a maximum matching in the compatibility graph of the $d$-splits consists of $p$ edges, then $q-p$ is the minimum dimension of a rectilinear space in which $(X, d)$ embeds.

Proof. Consider the median graph $G$ generated by the integral space $(X, \delta)$ associated to $(X, d)$. The $d$-splits then extend to the half-spaces of $G$. Assume that $(X, d)$ embeds in $\mathbb{R}^{k}$; then $G$ embeds as well. Each hyperplane perpendicular to some axis of $\mathbb{R}^{k}$ induces a pair of complementary half-spaces of $G$. The converse also holds true because any such pair is determined by some edge $\{u, v\}$ of $G$ : embedded in $\mathbb{R}^{k}$, the $i$ th coordinates of $u$ and $v$ differ for some $i$, so that the hyperplane perpendicular to the $i$ th axis and separating these coordinates induces the given pair of complementary half-spaces. Thus, the ordered set of all $d$-half-spaces of $(X, d)$ can be covered by $k$ pairs of "complementary" chains of $d$-half-spaces. Since $(X, d)$ has depth at most 2 , each chain consists of no more than two $d$-half-spaces. Therefore we obtain a matching of size $p=q-k$ in the compatibility graph of the $d$-splits. Conversely, any such matching leads to a covering of the $d$-halfspaces by $k$ pairs of "complementary" chains, thus providing an embedding in $\mathbb{R}^{k}$.

Notice that depth may increase when passing to subspaces. The following space and all of its maximal proper subspaces, however, have depth 2 . Let $(X, d)$ be the metric space given by the Cartesian product of two complete graphs with $2 t+1$ vertices each $(t \geq 1)$. The corresponding integral space $(X, \delta)$ with $\delta=2 d$ embeds in the product of two stars with $2 t+1$ end vertices each. $(X, d)$ has $4 t+2$ distinct $d$-splits, the compatibility graph of which is a disjoint union of two complete graphs of equal size. A maximum matching in this graph then consists of $2 t$ edges. Since the depth of $(X, d)$ is evidently $2,(X, d)$ embeds in $\mathbb{R}^{2 t+2}$ but not in $\mathbb{R}^{2 t+1}$. Removing a single point $x$ from $X$ solely affects the two $d$-half-spaces intersecting only in $x$ and their complements (in that the corresponding two $d$-splits become comparable). The resulting subspace also has depth 2 , and its comparability graph has an additional edge. This edge can now be used to obtain a perfect matching (with $2 t+1$ edges), so that the subspace embeds in $\mathbb{R}^{2 t+1}$. Therefore in order to decide embeddability in $\mathbb{R}^{k}$ (where $k=2 t+1$ ) we must check the subspaces having at least $k^{2}$ points. This establishes our claim that $c(k) \geq k^{2}$ for $k \geq 3$ odd. The proof of the single case $c(3) \geq 10$ is also based on Lemma 4 and makes use of the Cartesian product of two complete graphs with two and five vertices, respectively. The quadratic bound might still be far too optimistic; although we are confident that $c(k)$ exists for all $k$, we do not believe that its growth is actually bounded by a polynomial in $k$.

Interestingly, this problem with rectilinear embeddability has its counterparts for elliptic space: there is a seven-point condition for embeddability in the elliptic plane [6], [9], but the status of even the three-dimensional case remains open; see [10]. In contrast, $(k+3)$-point criteria are known to exist for embedding in $k$-dimensional Euclidean, spherical, and hyperbolic spaces, respectively [8], [5]. 


\section{Acknowledgment}

We would like to thank J. J. Seidel for encouraging us actually to list all forbidden minors.

\section{References}

1. H.-J. Bandelt, Generating median graphs from Boolean matrices, in $L_{1}$-Statistical Analysis and Related Methods (Y. Dodge, ed.), Elsevier/North-Holland, 1992, pp. 305-309.

2. H.-J. Bandelt and A. W. M. Dress, A canonical decomposition theory for metrics on a finite set, $A d v$. in Math. 92 (1992), 47-105.

3. H.-J. Bandelt and M. van de Vel, Embedding topological median algebras in products of dendrons, Proc. London Math. Soc. (3) 58 (1989), 439-453.

4. H.-J. Bandelt and M. van de Vel, Superextensions and the depth of median graphs, J. Combin. Theory Ser. A 57 (1991), 187-202.

5. L. M. Blumenthal, Theory and Applications of Distance Geometry, Oxford University Press, Oxford, 1953.

6. J. Haantjes and J. J. Seidel, The congruence order of the elliptic plane, Nederl. Akad. Wetensch. Indag. Math. 9 (1947), 403-405.

7. S. M. Malitz and J. I. Malitz, A bounded compactness theorem for $L^{1}$-embeddability of metric spaces in the plane, Discrete Comput. Geom. 8 (1992), 373-385

8. K. Menger, Untersuchungen über allgemeine Metrik, Math. Ann. 100 (1928), 75-163.

9. J. J. Seidel, De congruentie-orde van het elliptische vlak, Dissertation, Leiden, 1948.

10. J. J. Seidel, Metric problems in elliptic geometry, in The Geometry of Metric and Linear Spaces (L. M. Kelly, ed.), Lecture Notes in Mathematics, Vol. 490, Springer-Verlag, Berlin, 1975, pp. 32-43.

11. M. van de Vel, Matching binary convexities, Topology Appl. 16 (1983), 207-235.

12. M. L. J. van de Vel, Theory of Convex Structures, Elsevier/North-Holland, Amsterdam, 1993.

Received April 4, 1994, and in revised form December 15, 1994. 